BNL-HET-06/19

\title{
Signature of heavy Majorana neutrinos at a linear collider: Enhanced charged Higgs pair production
}

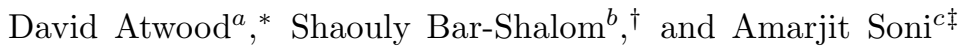 \\ ${ }^{a}$ Department of Physics and Astronomy, Iowa State University, Ames, IA 50011, USA \\ ${ }^{b}$ Physics Department, Technion-Institute of Technology, Haifa 32000, Israel \\ ${ }^{c}$ Theory Group, Brookhaven National Laboratory, Upton, NY 11973, USA
}

(Dated: May 24, 2018)

\begin{abstract}
A charged Higgs pair can be produced at an ee collider through a t-channel exchange of a heavy neutrino $(N)$ via $e^{+} e^{-} \rightarrow H^{+} H^{-}$and, if $\mathrm{N}$ is a Majorana particle, also via the lepton number violating (LNV) like-sign reaction $e^{ \pm} e^{ \pm} \rightarrow H^{ \pm} H^{ \pm}$. Assuming no a-priori relation between the effective $\mathrm{eNH}^{+}$coupling $(\xi)$ and light neutrino masses, we show that this interaction vertex can give a striking enhancement to these charged Higgs pair production processes. In particular, the LNV $H^{-} H^{-}$signal can probe $N$ at the ILC in the mass range $100 \mathrm{GeV} \lesssim m_{N} \lesssim 10^{4} \mathrm{TeV}$ and with the effective mixing angle, $\xi$, in the range $10^{-4} \lesssim \xi^{2} \lesssim 10^{-8}$ - well within its perturbative unitarity bound and the $\beta \beta_{0 \nu}$ limit. The lepton number conserving (LNC) $e^{+} e^{-} \rightarrow H^{+} H^{-}$mode can be sensitive to, e.g., an $\mathcal{O}(10) \mathrm{TeV}$ heavy Majorana neutrino at a $500 \mathrm{GeV}$ International Linear Collider (ILC), if $\xi^{2} \gtrsim 0.001$.
\end{abstract}

PACS numbers: 14.60.St,13.15.+g,13.66.Hk,12.15.Ji

The discovery of neutrino oscillations, which indicates mixing between massive neutrinos, stands as the first direct evidence for physics beyond the Standard Model (SM). Thus, the SM, or for that matter also the Minimal Supersymmetric Standard Model (MSSM) and any other multi-Higgs doublet model, has to be expanded to include massive neutrinos that mix. Since there is still no understanding of the nature of these massive neutrinos, i.e., Majorana or Dirac-like, the extensions to these models can go either way. However, from the theoretical point of view, the more natural and therefore more appealing way is to generate sub-eV Majorana neutrinos by adding heavy right-handed neutrino singlet fields ( $N$ with a mass $m_{N}$ ) and relying on the seesaw mechanism which yields $m_{\nu} \sim m_{D}^{2} / m_{N}$, where $m_{\nu}$ is the solar and atmospheric neutrino masses and $m_{D}$ are the Dirac mass terms. Thus, the classic seesaw mechanism links light neutrino masses $\left(m_{\nu} \sim 10^{-2} \mathrm{eV}\right)$ with new physics $\left(m_{N}\right)$ at the GUT scale (if $m_{D} \sim m_{W}$ ) or at the multi-TeV scale (if $m_{D} \sim m_{e}$ ) both are well motivated theoretically. In either case, this minimal seesaw setup yields a vanishingly small heavyto-light neutrino mixing: $U_{\ell N} \propto \sqrt{m_{\nu} / m_{N}}$, in which case heavy neutrinos effectively decouple.

In this work we will assume no a-priori relation between the couplings and light neutrino masses. Indeed, interesting models have been proposed, which assume that the underlying physics for sub-eV neutrino masses goes beyond the standard, minimal, seesaw framework (see e.g., 1, 2, 3, 4]), thus allowing the mixing angle $U_{\ell N}$ to depart from its classical seesaw value (i.e., $\sqrt{m_{\nu} / m_{N}}$ ). Our primary purpose in this paper is to propose col-

\footnotetext{
*Electronic address: atwood@iastate.edu

${ }^{\dagger}$ Electronic address: shaouly@physics.technion.ac.il

${ }^{\ddagger}$ Electronic address: soni@bnl.gov
}

lider experiments that can sensitively probe the relevant masses and mixing angles.

The fact that a Majorana mass term violates lepton number by two units, i.e. $\Delta L= \pm 2$, has striking phenomenological implications, some of which have been extensively studied in the past few decades - the most noticeable one being neutrinoless double beta decay $(A, Z) \rightarrow(A, Z+2)+e^{-}+e^{-}[5]$. With the upcoming CERN Large Hadron Collider (LHC) and the planned high-energy ee machines, the study of LNV collider signals in processes involving heavy Majorana neutrinos is particularly well motivated. Indeed, such high-energy LNV signals have been extensively studied in the past two decades: at $p p$ and $p \bar{p}$ collisions $6,67,8,9,10]$, at an $e^{+} e^{-}$[6, 11], $e^{-} e^{-}$12 and $e^{-} \gamma$ collisions [6, 13] and at an $e p$ machine [6, 11, 14]. In addition, LNV mediated by exchanges of a heavy Majorana $\mathrm{N}$ can be manifest in top-quark and W-boson decays [15] and in rare charged meson decays [8].

In this letter we propose that charged Higgs pair production at an ee machine like the ILC (International Linear Collider) can serve as a very sensitive probe of heavy neutrinos (N) with masses up to $m_{N} \sim 10^{4} \mathrm{TeV}$. The two processes we will explore are:

$$
\begin{aligned}
& e^{+} e^{-} \rightarrow H^{+} H^{-} \\
& e^{ \pm} e^{ \pm} \rightarrow H^{ \pm} H^{ \pm}
\end{aligned}
$$

These processes are generated at the tree level via a tchannel (and u-channel in the case of the like-sign $H^{ \pm} H^{ \pm}$ signal) exchange of a heavy $N$, which can have a sizable coupling to $e-H^{+}$when $m_{N}>>m_{W}$ (see below). Note also that, while the $\mathrm{H}^{+} \mathrm{H}^{-}$signal is not sensitive to the nature of $\mathrm{N}$, i.e., be it Majorana or Dirac particle, the like-sign $H^{ \pm} H^{ \pm}$signal is generated only if $\mathrm{N}$ is a Majorana neutrino. 


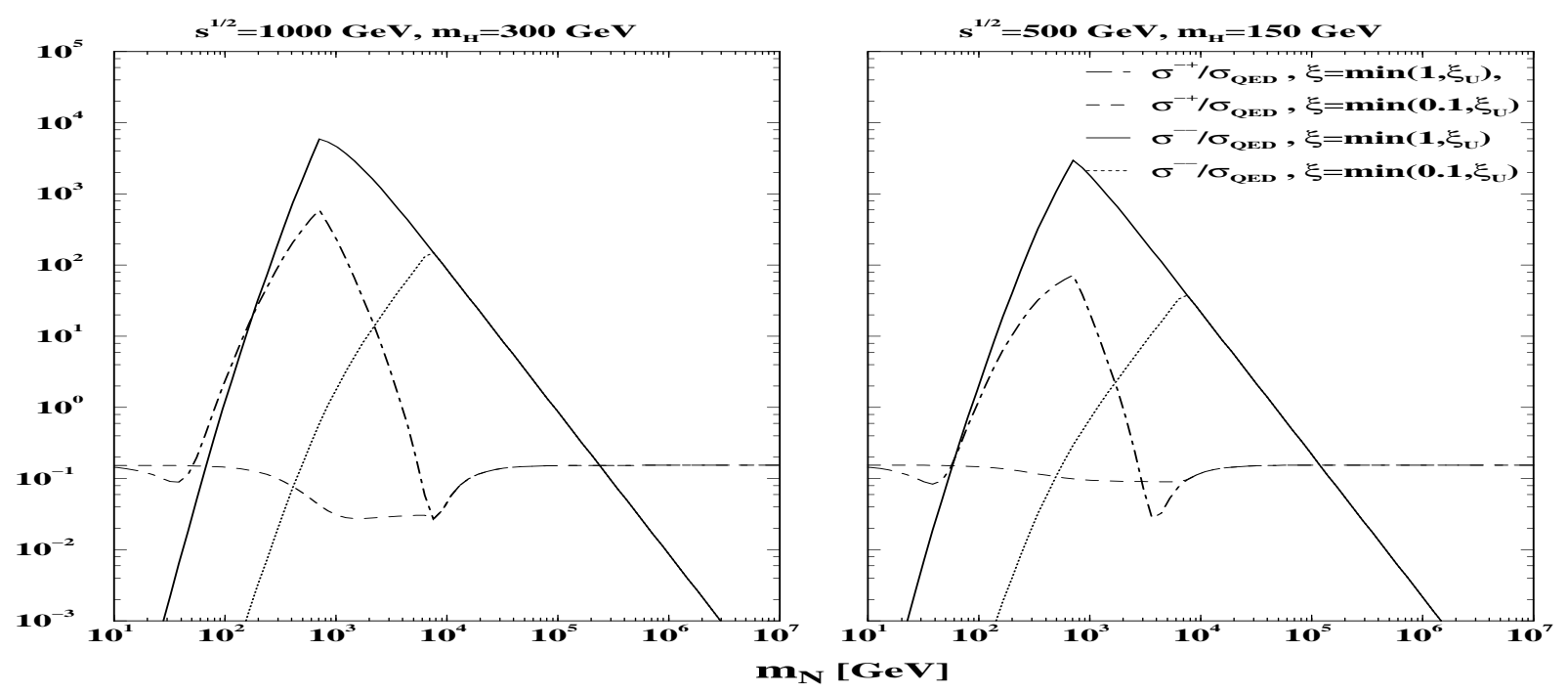

FIG. 1: The $L N V$ and $L N C$ cross-sections $\sigma^{--}$and $\sigma^{-+}$in units of $\sigma_{Q E D} \equiv \sigma\left(e^{+} e^{-} \rightarrow \gamma^{\star} \rightarrow \mu^{+} \mu^{-}\right)$, as a function of $m_{N}$ and with $\xi=\min \left(0.1, \xi_{U}\right)$ or $\xi=\min \left(1, \xi_{U}\right)$, for a $500 \mathrm{GeV}$ (right figure) and $1000 \mathrm{GeV}$ (left figure) ee collider with charged Higgs masses $m_{H}=150$ and 300 GeV, respectively.

In any model with more than one Higgs doublet, a right handed neutrino (be it Majorana or Dirac-like) can couple to a charged Higgs via its Yukawa interactions with the scalar doublets $Y L \Phi_{i} N$. The $H^{+} \ell N$ Yukawalike coupling can, therefore, be written as:

$$
\mathcal{L}_{H^{+} \ell N}=\frac{g}{\sqrt{2}} \bar{N}\left(f_{v}^{\ell} \frac{m_{\ell}}{m_{W}} R+f_{v}^{N} \frac{m_{D}}{m_{W}} L\right) \ell H^{+}+h . c .
$$

where $m_{D}$ and $m_{\ell}$ are the neutrinos and charged leptons Dirac mass terms, respectively, and the $f_{v}$ 's are some functions of the Higgs doublets VEV's. For example, $f_{v}^{\ell}=\left(f_{v}^{N}\right)^{-1}=\tan \beta$ within an extended supersymmetric or a non-supersymmetric type II two Higgs doublet model with heavy right handed neutrinos.

Since we will be interested in the effects of a heavy Majorana-type neutrino, we assume $N$ to have a Majorana mass term of the form $M N N / 2$. Thus, we rewrite the $H^{+} e N$ interaction Lagrangian (3) in a generic form, in terms of the physical mass of $N, m_{N}$, and assuming $f_{v}^{e} m_{e}<<f_{v}^{N} m_{D}$ :

$$
\mathcal{L}_{H^{+} e N}=\frac{g}{2 \sqrt{2}} \xi \frac{m_{N}}{m_{W}} \bar{N}\left(1-\gamma_{5}\right) e H^{+}+\text {h.c. },
$$

where the dimensionless parameter $\xi$ defines an effective $H^{+} e N$ coupling strength that will be the key factor which controls the size of the cross-section for reactions (1) and (2). In particular, we absorb in $\xi$ any relation between $m_{D}$ and $m_{N}$ which will depend on the mechanism responsible for the generation of neutrino masses. Depending on the size of $\xi$, the interaction term in (44) can have striking implications for $\mathrm{N}-\mathrm{H}^{+}$phenomenology at an highenergy ee collider, in particular, for the charged Higgs pair production mechanisms in (11) and (2).
As mentioned earlier, we will assume no a-priori relation between $m_{N}$ and light neutrino masses and mixing, thus, adopting a model independent approach towards the effective $\mathrm{H}^{+} e N$ coupling $\xi$, and treating it as a free parameter (assuming for simplicity that it is real). As such, $\xi$ should be subject only to existing experimental constraints and perturbative unitarity if applicable (see below). However, since there is no direct experimental constraint on $\xi$ that we know of, we will use the seesawlike framework as a guide for estimating the experimentally allowed size of $\xi$. In particular, within a seesawlike mechanism for generating light neutrino masses, the Dirac mass $m_{D}$ is related to the physical heavy Majorana mass by $m_{D} \sim U_{\ell N} m_{N}$, where $U_{\ell N} \sim m_{D} / m_{N}$ being the heavy-to-light neutrino mixing angle (see e.g., Pilaftsis in [4]). Thus, in this case we obtain (see Eqs. 3 and [4):

$$
\xi \equiv f_{v}^{N} \cdot U_{e N}
$$

It should be emphasized again that, although the minimal seesaw framework restricts the mixing $U_{\ell N}$ to be vanishingly small, one cannot rule out $U_{\ell N} \sim \mathcal{O}(1)$ even for $m_{N} \sim \mathcal{O}(100) \mathrm{GeV}$, based on seesaw-like scenarios which go beyond the minimal framework, such as radiative seesaw, double seesaw and fine-tuned relations or symmetries between the mass matrices $m_{D}$ and $m_{N}$, as was noted for example in [1, 2, 3, ,4].

Using the seesaw-like motivated relation in (5), the existing experimental constraints on $U_{e N}$ can be applied as an estimate for the allowed size of $\xi$. In particular, $U_{e N}$ is constrained from precision electroweak data (e.g., from invisible Z-decays) [16]: $\left|U_{e N}\right|^{2} \leq 0.012$ at $90 \% \mathrm{CL}$ if $m_{N}>m_{Z}$. This does not rule out $\bar{\xi} \sim \mathcal{O}(1)$, since $f_{v}^{N}$ which depends on the details of the scalar sector of the underlying theory, can be of order 10. A stronger con- 
straint on $U_{e N}$ comes from neutrinoless double- $\beta$ decay $\left(\beta \beta_{0 \nu}\right)$ [17]: $\sum_{N} U_{e N}^{2} / m_{N} \leq 5 \cdot 10^{-5} \mathrm{TeV}^{-1}$. We note though, that the $\beta \beta_{0 \nu}$ bound, although tighter, is subject to cancelations if there are more than one heavy N's that couple to an electron, in particular if they have different masses and mixings (see e.g., discussion by G. Belanger et al. in [12]).

As noted above, another constraint can be applied on the $\xi-m_{N}$ plane from perturbative unitarity by demanding that $\Gamma_{N}<m_{N} / 2$. In particular, in our simplified framework $N$ can decay via: $N \rightarrow$ $W^{\mp} \ell^{ \pm}, Z \nu_{\ell}, H \nu_{\ell}, H^{\mp} \ell^{ \pm}$, where the decay rates into the gauge-bosons is $\propto\left|U_{e N}\right|^{2}$, see e.g., 13]. Taking $M_{N}^{2} \gg>m_{W}^{2}, m_{Z}^{2}, m_{H^{0}}^{2}, m_{H^{+}}^{2}$, the total decay width of $N$ (i.e., summing over the above decay channels) is:

$$
\Gamma_{N} \approx 3|\xi|^{2} \Gamma_{N}^{0} \cdot\left(1+\frac{1}{\left|f_{v}^{N}\right|^{2}}\right) ; \Gamma_{N}^{0} \equiv \frac{g^{2}}{64 \pi} \frac{m_{N}^{2}}{m_{W}^{2}} m_{N}
$$

Thus, taking $\left|f_{v}^{N}\right|^{2}>>1$ (i.e., assuming that the total $\mathrm{N}$ width is dominated by its decays to the Higgs-bosons), perturbative unitarity imposes the following bound: ${ }^{[1]}$

$$
\xi \lesssim \xi_{U} \sim \frac{4}{g} \sqrt{\frac{2 \pi}{3}} \cdot \frac{m_{W}}{m_{N}} \sim 0.715 \frac{T e V}{m_{N}}
$$

This gives e.g., $\xi \lesssim 1$ for $m_{N} \gtrsim 700 \mathrm{GeV}$ or $\xi \lesssim 0.1$ for $m_{N} \gtrsim 7 \mathrm{TeV}$. In what follows, we will always apply the unitarity bound in (7), or restrict $\xi$ to be smaller than either 1 or 0.1 when the unitarity bound does not apply.

The cross-section for the LNV $e^{-} e^{-} \rightarrow H^{-} H^{-}$mode is given by:

$$
\sigma^{--}=\sigma_{Q E D} \cdot \xi^{4} \frac{3 \beta}{32 s_{W}^{4}} \frac{r_{N}^{3}}{r_{W}^{2}}\left(\frac{2}{w^{2}-\beta^{2}}+\frac{\ln \left(\frac{w+\beta}{w-\beta}\right)}{\beta w}\right),
$$

where $\sigma_{Q E D} \equiv \sigma\left(e^{+} e^{-} \rightarrow \gamma^{\star} \rightarrow \mu^{+} \mu^{-}\right)=4 \pi \alpha^{2} / 3 / s$, $s_{W} \equiv \sin \theta_{W}, \beta \equiv \sqrt{1-4 r_{H}}, w \equiv 1+2 r_{N}-2 r_{H}$ and $r_{x} \equiv m_{x}^{2} / s$. Thus, in the limit $m_{N}^{2}>>s$, we obtain $\sigma^{--} \propto \xi^{4} m_{N}^{2} / m_{W}^{4}$, i.e., the cross-section grows with $m_{N}$ for a constant effective mixing $\xi$. This growth behavior is, however, "cured" when we impose the perturbative unitarity bound in (7):

$$
\left.\sigma^{--}\left(m_{N}^{2}>>s\right)\right|_{\xi=\xi_{U}} \sim \frac{32 \pi \beta}{9} \frac{1}{m_{N}^{2}} .
$$

In Fig. 1 we plot the LNV cross-section $\sigma^{--}$in units of $\sigma_{Q E D}$, as a function of $m_{N}$ for both a 500 and 1000

[1] We note that partial wave unitarity of $e^{-} e^{-} \rightarrow H^{-} H^{-}$also sets a bound on $\xi$ which is, however, weaker than $\xi_{U}$ coming from $\Gamma_{N}<m_{N} / 2$. We have also checked that the measured $g-2$ of the electron does not further constrain $\xi$.
$\mathrm{GeV}$ ee machines. For illustration, we take $m_{H^{+}}=150$ and $300 \mathrm{GeV}$, for the $\sqrt{s}=500$ and $1000 \mathrm{GeV}$ colliders, respectively. We consider the cases $\xi=0.1$ and $\xi=1$, imposing the unitarity bound such that $\xi=\min \left(0.1, \xi_{U}\right)$ and $\xi=\min \left(1, \xi_{U}\right)$, respectively (recall that the unitarity bound implies $\xi_{U} \sim 0.1$ already for $m_{N} \sim 7 \mathrm{TeV}$ ). As expected, there is a sharp decrease in $\sigma^{--}$which occurs once $\xi$ enters the unitarity bound regime $\left(\xi \rightarrow \xi_{U}\right)$, for which the cross-section drops as $m_{N}^{-2}$ as seen in (9).

In the mass range $100 \mathrm{GeV} \lesssim m_{N} \lesssim$ few $\cdot 10^{3} \mathrm{TeV}$, despite fixing $\xi=\min \left(0.1, \xi_{U}\right)$ due to unitarity, we still see that $\sigma^{--} \sim 10^{-3} \cdot \sigma_{Q E D}$. Thus, recalling that any given design of an ee machine relies on having 10000 events per unit $\sigma_{Q E D}$, one expects more than $10 e^{-} e^{-} \rightarrow H^{-} H^{-}$ events even if $m_{N} \sim 10^{3}-10^{4} \mathrm{TeV}$. In Fig. 2 we plot a "naive" discovery limit (demanding 10 events for discovery) for $e^{-} e^{-} \rightarrow H^{-} H^{-}$in the $m_{N}-\xi^{2}$ plane, at an ILC with $\sqrt{s}=500 \mathrm{GeV}, 1 \mathrm{TeV}$ and $3 \mathrm{TeV}$, setting $m_{H}=150,300$ and $600 \mathrm{GeV}$, respectively, and with an integrated luminosity of $L=100 \times(\sqrt{s} / \mathrm{TeV})^{2} \mathrm{fb}^{-1}$ which corresponds to $L \cdot \sigma_{Q E D} \sim 10000$ at each c.m. energy. We see that the like-sign Higgs pair production signal is indeed striking at an ILC; it is potentially observed even if $m_{N} \sim 10^{4} \mathrm{TeV}$ and $\xi^{2} \sim 10^{-8}$ - an extremely small value for the effective neutrino mixing angle which is consistent with the unitarity bound and more than 7 orders of magnitudes smaller than the $\beta \beta_{0 \nu}$ bound at $m_{N} \sim 10^{4}$ $\mathrm{TeV}$ !

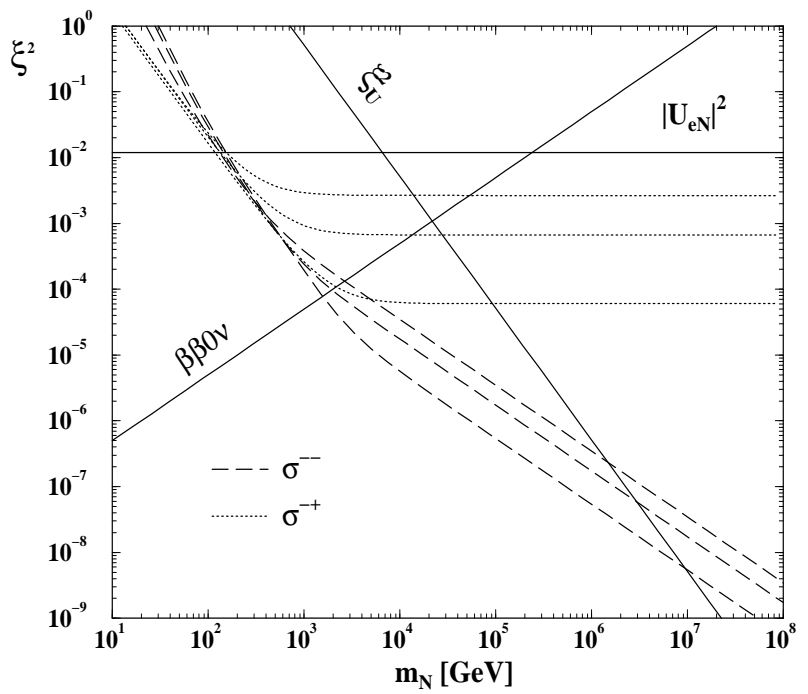

FIG. 2: Discovery limit for $e^{-} e^{-} \rightarrow H^{-} H^{-}$(long-dashed lines) and for $e^{-} e^{+} \rightarrow H^{-} H^{+}$(dotted lines), at an ILC with $\sqrt{s}=500 \mathrm{GeV}$ (upper line), $\sqrt{s}=1 \mathrm{TeV}$ (middle line) and $\sqrt{s}=3 \mathrm{TeV}$ (lower line), see also text. The parameter space above the lines corresponds to the observable events. Also plotted (solid lines): the experimental limits on $U_{e N}^{2}$ from $\beta \beta_{0 \nu}$ and on $\left|U_{e N}\right|^{2}$ from invisible Z-decays, as well as the perturbative unitarity bound from (7). Here the parameter space above the lines are ruled out.

Let us now turn to the lepton number conserving (LNC) process $e^{+} e^{-} \rightarrow H^{+} H^{-}$. There are 3 diagrams 
contributing to this process: the two "standard" (i.e., within multi-Higgs models) s-channel $\gamma$ and $Z$ exchanges and a 3rd t-channel $\mathrm{N}$ exchange one. The cross-section $\sigma^{-+} \equiv \sigma\left(e^{-} e^{+} \rightarrow H^{-} H^{+}\right)$can be decomposed accordingly as $\sigma^{-+} \equiv \sigma_{S}^{-+}+\sigma_{S N}^{-+}+\sigma_{N}^{-+}$, where the subscripts $S, N$ and $S N$ denote the standard s-channel $\gamma, Z$ contribution, the t-channel $N$ contribution and the interference between the standard and the $N$-exchange diagrams. While $\sigma_{S}$ can be found in the literature [18], the new $N$ exchange contributions to the total cross-section are:

$$
\begin{aligned}
& \sigma_{S N}^{-+}=C_{N} \frac{1-4 r_{Z} s_{W}^{2} c_{W}^{2}}{c_{W}^{2}\left(1-r_{Z}\right)}\left[\ln \left(\frac{w+\beta}{w-\beta}\right)-\frac{\beta w}{2 v}\right], \\
& \sigma_{N}^{-+}=C_{N} \frac{2 \xi^{2}}{w^{2}-\beta^{2}} \frac{r_{N}}{r_{W}}\left[w \ln \left(\frac{w+\beta}{w-\beta}\right)-2 \beta\right],(10)
\end{aligned}
$$

where $v \equiv r_{N}+(1-w)^{2} / 4$ and

$$
C_{N} \equiv \sigma_{Q E D} \cdot \xi^{2} \frac{3 v}{32 s_{W}^{4}} \frac{r_{N}}{r_{W}} .
$$

For a constant $\xi$ and in the limit $m_{N}^{2}>>s$, both $\sigma_{S N}^{-+}$and $\sigma_{N}^{-+}$approach a constant with respect to $m_{N}$. As $\xi$ enters the perturbative unitarity bound regime, i.e., $\xi \rightarrow \xi_{U} \sim 1 / m_{N}$, the interference term dominates since $\sigma_{S N}^{-+} / \sigma_{N}^{-+} \propto m_{N}^{2} / s$, but still drops with $m_{N}$ as $\sigma_{S N}^{-+} \propto 1 / m_{N}^{2}$. Thus, in this large $N$ mass range the total $H^{+} H^{-}$cross-section converges to the standard value: $\sigma^{-+}\left(m_{N}^{2}>>s, \xi=\xi_{U}\right) \rightarrow \sigma_{S}^{-+}$. This behavior can be seen in Fig. 1, where we have plotted $\sigma^{-+}$in units of the point cross-section $\sigma_{Q E D}$, as a function of $m_{N}$ for both a 500 and $1000 \mathrm{GeV}$ ee machines, for which we take $m_{H^{+}}=150$ and $300 \mathrm{GeV}$, respectively. As in the LNV $H^{-} H^{-}$case, we consider $\xi=0.1$ and $\xi=1$, imposing the unitarity bound such that $\xi=\min \left(0.1, \xi_{U}\right)$ and $\xi=\min \left(1, \xi_{U}\right)$.

Note that around $m_{N} \sim \sqrt{s}$ the t-channel $\mathrm{N}$ exchange in $H^{+} H^{-}$production can become sizable. We, thus, define the number of standard deviations $\left(N_{S D}^{N}\right)$ with which the t-channel $N$ exchange in $e^{-} e^{+} \rightarrow H^{-} H^{+}$can be detected at an ILC:

$$
N_{S D}^{N}=\frac{\sqrt{L}\left(\sigma^{-+}-\sigma_{S}^{-+}\right)}{\sqrt{\sigma^{-+}}} .
$$

In Fig. 2 we plot the $5 \sigma\left(N_{S D}^{N}=5\right)$ discovery contours of the $N$-exchange contribution to $e^{-} e^{+} \rightarrow H^{-} H^{+}$in the $\xi^{2}-m_{N}$ plane. As in the $H^{-} H^{-}$case, we take an ILC with $\sqrt{s}=500 \mathrm{GeV}, 1 \mathrm{TeV}$ and $3 \mathrm{TeV}$ (setting $m_{H}=$ 150,300 and $600 \mathrm{GeV}$, respectively), and an integrated luminosity of $L=100 \times(\sqrt{s} / \mathrm{TeV})^{2} \mathrm{fb}^{-1}$. Although not as promising as the LNV $H^{-} H^{-}$signal, we see that the $\mathrm{N}$-mediated $\mathrm{H}^{-} \mathrm{H}^{+}$LNC signal can be detected at $5 \sigma$ in an ILC with e.g., $\sqrt{s}=1(3) \mathrm{TeV}$, for a very heavy $N$ with a mass in the range $10(2) \mathrm{TeV} \lesssim m_{N} \lesssim 30(100) \mathrm{TeV}$ and $\xi^{2} \sim 6 \cdot 10^{-4}\left(6 \cdot 10^{-5}\right)$ - a value well within the $\beta \beta_{0 \nu}$ bound and consistent with the unitarity bound.

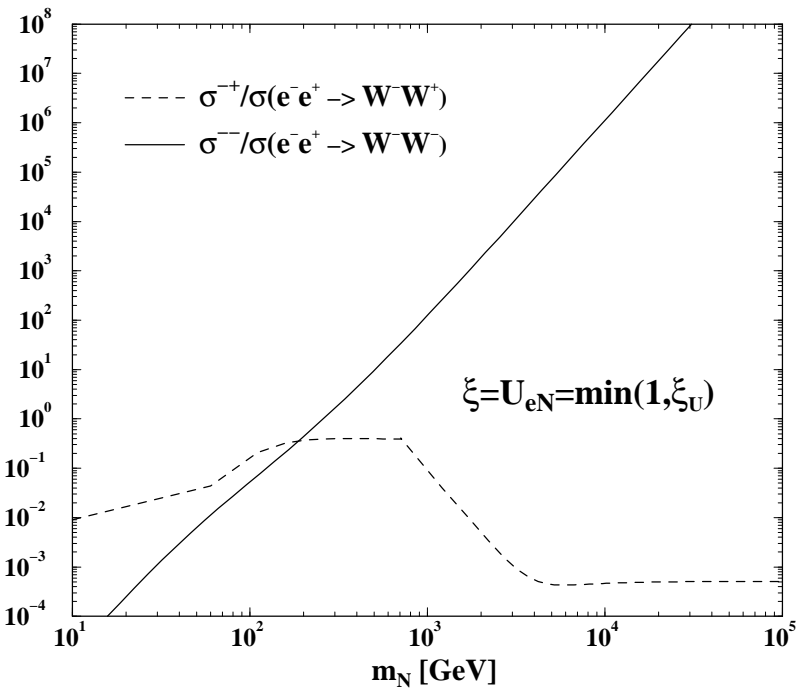

FIG. 3: The cross-sections ratios $\sigma^{--} / \sigma\left(e^{-} e^{-} \rightarrow W^{-} W^{-}\right)$ and $\sigma^{-+} / \sigma\left(e^{-} e^{+} \rightarrow W^{-} W^{+}\right)$, as a function of $m_{N}$, for $\xi=$ $U_{e N}=\min \left(1, \xi_{U}\right)$ at a $500 \mathrm{GeV} I L C$.

Admittedly, our criteria for a $5 \sigma$ discovery (or 10 events in the $H^{-} H^{-}$case), as plotted in Fig. 2, is rather naive since we do not take into account the possible charged Higgs decays, their branching ratios and the corresponding detection efficiencies. Nonetheless, this simplified discovery criteria allows one to get an idea of the rather striking prospects of these charged Higgs pair production signals (especially the LNV $H^{-} H^{-}$one), as potential probes of heavy Majorana neutrinos. In particular, to further appreciate the significance of both the LNV and LNC Higgs pair production signals, we plot in Fig. 3 the ratios $\sigma^{--} / \sigma\left(e^{-} e^{-} \rightarrow W^{-} W^{-}\right)$and $\sigma^{-+} / \sigma\left(e^{-} e^{+} \rightarrow W^{-} W^{+}\right)$at a $500 \mathrm{GeV}$ ILC, ${ }^{[2],[3]}$ as a function of $m_{N}$ and for $\xi=U_{e N}=\min \left(1, \xi_{U}\right)$, i.e., assuming $f_{v}^{N}=1$.

Take for example the $H^{-} H^{-}$signal for which we get $\sigma^{--} / \sigma\left(e^{-} e^{-} \rightarrow W^{-} W^{-}\right)>10$ already for $m_{N} \gtrsim \sqrt{s}$ $(=500 \mathrm{GeV})$. Recall that $\sigma\left(e^{-} e^{-} \rightarrow W^{-} W^{-}\right) \propto U_{e N}^{4}$ is the inverse $\beta \beta_{0 \nu}$ (t-channel $\mathrm{N}$ exchange) process, which was extensively studied as a potential discovery channel for a heavy $N$ at an ILC 12], and which stands as the leading background to the $e^{-} e^{-} \rightarrow H^{-} H^{-}$process. Thus, the $H^{-} H^{-}$signal should be easily detected at a $500 \mathrm{GeV}$ ILC if $m_{N} \gtrsim 500 \mathrm{GeV}$. Clearly, if $m_{H}>m_{t}+m_{b}$, then the $H^{-} H^{-}$signal can be searched for via $e^{-} e^{-} \rightarrow H^{-} H^{-} \rightarrow b b \overline{t t}$. In this case the effective $H^{-} H^{-} / W^{-} W^{-}$ratio is further (dramatically) enhanced by a $(2 \rightarrow 2) /(2 \rightarrow 4)$ phase-space factor. As for the LNC $H^{-} H^{+}$channel, we get $\sigma^{-+} / \sigma\left(e^{-} e^{+} \rightarrow W^{-} W^{+}\right) \gtrsim 0.1$ for $100 \mathrm{GeV} \lesssim m_{N} \lesssim 1000 \mathrm{GeV}$. Thus, here also, in particular if $m_{H}>m_{t}+m_{b}$, the N-exchange contribution in the $\mathrm{H}^{-} \mathrm{H}^{+}$channel should be easily accessible to a 500 $\mathrm{GeV}$ ILC if $m_{N} \sim \mathcal{O}(1) \mathrm{TeV}$, even if $\xi^{2}<10^{-2}$ - see also Fig. 2,

To summarize, we have examined the potential role 
of the charged Higgs pair production processes $e^{-} e^{+} \rightarrow$ $H^{-} H^{+}$and $e^{-} e^{-} \rightarrow H^{-} H^{-}$at an ILC, as possible probes of new dynamics mediated by heavy neutrinos, $\mathrm{N}$. We have shown that the $m_{N} / m_{W}$ enhancement factor in the $e-N-H^{+}$interaction vertex (i.e., compared to the $e-N-W^{+}$vertex) makes these processes extremely sensitive to the t-channel $N$-exchange that drives them. In particular, we find that the LNV $\mathrm{H}^{-} \mathrm{H}^{-}$signal is sensitive to a heavy neutrino with a mass up to $10^{4} \mathrm{TeV}$ and an effective Yukawa-like coupling to an electron as small as $\xi \sim 10^{-4}$. For example, in a supersymmetric non-minimal seesaw-like framework with heavy Majorana neutrinos, for which $\xi=U_{e N} \cdot f_{v}^{N}$ and $f_{v}^{N}=1 / \tan \beta$, one expects $\xi=U_{e N} / \tan \beta$, where $U_{e N}$ is the $\nu_{e}-N$ mixing angle. Thus, taking $\tan \beta \sim \mathcal{O}(30)$ or $\tan \beta \sim \mathcal{O}(3)$, the LNV $H^{-} H^{-}$signal can probe a very heavy $N$ with $m_{N} \sim 10^{4} \mathrm{TeV}$ for the very small values of the neutrino mixing angles $U_{e N}^{2} \sim 10^{-5}$ or $U_{e N}^{2} \sim 10^{-7}$, respectively. This striking result is compatible with both the $\beta \beta_{0 \nu}$ and the perturbative unitarity bounds on the $U_{e N}^{2}-m_{N}$ plane and, to the best of our knowledge, stands out as an exceptional signal of heavy Majorana neutrinos at large colliders.

In the case of the LNC signal $e^{+} e^{-} \rightarrow H^{+} H^{-}$, we find that an ILC with a center of mass energy of $1 \mathrm{TeV}$, can potentially probe a heavy Majorana with a mass $m_{N} \sim$ $\mathcal{O}(10) \mathrm{TeV}$, if $\xi^{2} \sim 0.001$ - also within the $\beta \beta_{0 \nu}$ and the [2] We have included the contribution from the t-channel $\mathrm{N}$ exchange diagram in $\sigma\left(e^{-} e^{+} \rightarrow W^{-} W^{+}\right)$.

[3] Following suggestions by Tao Han, we have also considered the constraints on $U_{e N}$ from the LEP2 measurements of $\sigma\left(e^{-} e^{+} \rightarrow\right.$ perturbative unitarity bounds on the $U_{e N}^{2}-m_{N}$ plane.

Finally, in passing, we note that recently an interesting model that can be tested at the ILC through our charged Higgs pair production mechanisms was suggested by $\mathrm{Ma}$ in [3]. In Ma's model a second scalar doublet $\left(\eta^{+}, \eta^{0}\right)$ is introduced and the light neutrinos acquire sub-eV masses through a loop involving both $N$ and $\eta^{0}$ - a radiative seesaw mechanism. As a consequance, the coupling $e-$ $N-\eta^{+}$is much less constrained (than in the minimal seesaw scenario) and should, therefore, be studied at the ILC via the reaction $e^{-} e^{-} \rightarrow \eta^{-} \eta^{-}$, where each of the $\eta^{-}$'s in the final state can further decay via $\eta^{-} \rightarrow \eta^{0} W^{-}$, $\eta^{0}$ being the $\mathrm{SU}(2)$ partner of $\eta^{+}$. It is also worth noting that, in this model, $\eta^{0}$ can be the lightest stable particle due to an exact $Z_{2}$ symmetry of the model, making it a good candidate for dark matter [3]. In such a case, the reaction $e^{-} e^{-} \rightarrow \eta^{-} \eta^{-}$will lead to a unique signal of two same-charge $W$-bosons and a missing energy carried by the two $\eta^{0}$ 's.

We thank Gad Eilam, Tao Han, Ernest Ma and Jose Wudka for very useful discussions. S.B.S thanks the hospitality of the theory group in Brookhaven National Laboratory where part of this study was performed. This work was supported in part by US DOE Contract Nos. DE-FG02-94ER40817 (ISU) and DE-AC02-98CH10886 (BNL).

$W^{-} W^{+}$) [19]. We found that $e^{-} e^{+} \rightarrow W^{-} W^{+}$is not sensitive at LEP2 to the N-exchange diagram if $U_{e N} \lesssim 0.1$, i.e., within its bound from invisible $\mathrm{Z}$ decays.
[1] For a recent overview on non-minimal seesaw-like models see, J. W. F. Valle, J. Phys. Conf. Ser. 53, 473 (2006).

[2] F. del Aguila and M. Zralek, Acta Phys. Polon. B33, 2585 (2002); F. del Aguila, M. Masip and J. L. Padilla, Phys. Lett. B627, 131 (2005).

[3] E. Ma, Phys. Rev. D73, 077301 (2006).

[4] A. Pilaftsis, Z. Phys. C55, 275 (1992) (also in hep-ph/9901206); D. Tommasini it et al, Nucl. Phys. B444, 451 (1995); J. Gluza Acta Phys. Polon. B33, 1735 (2002).

[5] For recent reviews see e.g.: A. S. Barabash, JINST 1, P07002 (2006); P. Vogel, Prog. Part. Nucl. Phys. 57, 177 (2006).

[6] F. del Aguila, J.A. Aguilar Saavedra and R. Pittau, J. Phys. Conf. Ser. 53, 506 (2006).

[7] H. Tso-Hsiu, C. Cheng-Rui and T. Zhi-Jian, Phys. Rev. D42, 2265 (1990); D.A. Dicus and D.D. Karatas, Phys. Rev. D44, 2033 (1991). A. Datta, M. Guchait and D.P. Roy, Phys. Rev. D47, 961 (1993); A. Ferrari et al., Phys. Rev. D62, 013001 (2000).

[8] A. Ali, A.V. Borisov and N.B. Zamorin, Eur. Phys. J. bf C21, 123 (2001).

[9] F.M.L. Almeida et al., Phys. Rev. D62, 075004 (2000).
[10] T. Han and B. Zhang, Phys. Rev. Lett. 97, 171804 (2006).

[11] W. Buchmüller and C. Greub, Nucl. Phys. B363, 345 (1991); F.M.L. Almeida et al., Eur. Phys. J. C30, 327 (2003).

[12] T.G. Rizzo, Phys Let. B116, 23 (1982) and hep-ph/9501261. G. Belanger et al., Phys. Rev. D53, 6292 (1996).

[13] S. Bray, J.S. Lee and A. Pilaftsis, Phys. Lett. B628, 250 (2005).

[14] M. Flanz, W. Rodejohann and K. Zuber, Phys. Lett. B473, 324 (2000), Erratum-ibid. B480, 418 (2000); A. Ali, A.V. Borisov and D.V. Zhuridov, hep-ph/0512005

[15] S. Bar-Shalom et al., Phys. Lett. B643, 342 (2006).

[16] S. Bergmann and A. Kagan, Nucl. Phys. B538, 368 (1999).

[17] For a recent analysis of the $\beta \beta_{0 \nu}$ bound see e.g., P. Benes et al, Phys. Rev. D71, 077901 (2005).

[18] See e.g., S. Komamiya, Phys. Rev. D38, 2158 (1988); A. Djouadi et al., Z. Phys. C74, 93 (1997).

[19] See e.g., The LEP Collaborations and the LEP Electroweak Working Group, hep-ex/0612034. 